\title{
Apports de la pédagogie Montessori à l'apprentissage du lexique des enfants non-lecteurs de Grande Section
}

\author{
Contributions of Montessori method in the learning of vocabulary by \\ non-reader children at preschool
}

\section{Aportes de la pedagogía Montessori en el aprendizaje del léxico de niños no lectores de preescolar}

\author{
María EMILIA Bonilla DuRÁN \\ Colegio Nuestra Señora de Sion, Costa Rica \\ Patricia Mora BarRantes \\ Amazon, Costa Rica \\ Marisol Rojas Díaz \\ Alianza Francesa, Costa Rica
}

\section{Résumé}

Même si la méthode Montessori n'était pas conçue - à l'origine - pour l'enseignement des langues étrangères, nous considérons que ses principes universels de formation de l'enfant peuvent aussi être appliqués à la classe de Français langue étrangère.

L'objectif général a été d'initier les enfants non-lecteurs de deux groupes de Grande Section de l'École Saint Benedict à la compréhension de l'album "Petit chat perdu " au travers des principes montessoriens. Quant à la méthodologie, nous avons opté pour une approche qualitative. Nous avons utilisé deux instruments : l'interview et des observations participantes et non participantes. Pour la collecte des données nous avons employé une grille d'observation et un journal de bord.

Le point de départ a consisté à tirer des apports utiles de la théorie Montessori pour le cours de français. Ensuite, nous avons mis en pratique une séquence pédagogique qui favorise la compréhension orale avec des activités variées inspirées de cette pédagogie 
tout en respectant les principes de la théorie. Enfin, nous avons analysé les avantages et les inconvénients de cette adaptation pour initier des enfants non-lecteurs à l'apprentissage du FLE.

Mots clés : français langue étrangère, la méthode Montessori, compréhension orale, enfants non-lecteurs

\begin{abstract}
Even if the Montessori method was not originally designed for teaching foreign languages, we consider that its universal principles of child development can also be applied to the teaching of French as a Foreign Language.

The general objective was to initiate non-reader children of two preschool classes from Saint Benedict School, to the understanding of the book "Petit Chat Perdu" through Montessorian principles. As for the methodology, we opted for a qualitative approach. We used two instruments: the interview and participant and non-participant observations. For the data collection we used an observation guide and a logbook.

The starting point was to draw useful contributions from the Montessori theory for the French course. Then, we put into practice a learning sequence that promotes oral comprehension with multiple activities inspired by this method, while respecting the principles of the theory. Finally, we analyzed the advantages and disadvantages of this adaptation in the introduction of French as Foreign Language to non-reader children.
\end{abstract}

Keywords: French as Foreign Language, Montessori method, oral comprehension, non-reader children

\title{
Resumen
}

Incluso si el método Montessori no fue concebido originalmente para la enseñanza de lenguas extranjeras, consideramos que sus principios universales de formación de los niños y las niñas pueden ser aplicados igualmente para la clase de francés como lengua extranjera. El objetivo de la investigación fue iniciar a los niños y las niñas no lectores de dos grupos de preescolar de la Escuela Saint Benedict en la comprensión del cuento "Petit chat perdu" a través de los principios montessorianos. En cuanto a la metodología, se optó por una perspectiva cualitativa. Se utilizaron dos instrumentos: la entrevista y las observaciones de participantes y no participantes. Para la recolección de los datos se emplearon una tabla de observación y un diario de campo.

El punto de partida consistió en extraer aportes útiles de la teoría Montessori para la clase de francés. Posteriormente, se puso en práctica una secuencia pedagógica que favorece la comprensión oral con actividades variadas inspiradas en la pedagogía respetando al mismo tiempo los principios de la teoría. Por último, se analizaron las ventajas e inconvenientes de dicha adaptación para iniciar a niñas y niños no lectores en el aprendizaje del francés como lengua extranjera.

Palabras clave: francés como lengua extranjera, método Montessori, comprensión oral, niños no lectores 


\section{Introduction}

L ors de son apparition en 1907, la méthode Montessori a bouleversé la vision traditionnelle d'apprentissage des enfants. Celle-ci propose une formation centrée sur les besoins et les intérêts de l'enfant, tout en respectant le rythme individuel de chaque apprenant, l'autogestion, l'auto-correction ainsi que la création d'un espace d'apprentissage soigneusement préparé.

En principe, la pédagogie Montessori n'a pas été conçue pour l'enseignement des langues. Cependant, ceci n'a pas empêché son application en cours de langue étrangère par des multiples éducateurs contemporains. Tel est le cas de l'établissement The English Montessori School à Madrid où on a réussi à stimuler l'écriture et la lecture en cours de langue, parallèlement à un apprentissage intégral de tous les autres cours (Mathématiques, Sciences naturelles, Histoire, etc.). Il s'agit d'une école britannique bilingue Montessori où les enfants sont accompagnés de guides et non pas par des " distributeurs de la connaissance " (un terme utilisé par la même école pour accentuer la différence).

Notre motivation a été de connaître si une méthode non conçue pour l'enseignement des langues pouvait-elle être utile pour l'enseignement du FLE aux enfants non-lecteurs. C'est pourquoi la recherche a été envisagée comme un " laboratoire d'expérimentation scientifique " en classe de FLE en créant une interaction entre : l'approche holistique de la philosophie Montessori, l'enseignement du FLE et la littérature enfantine. Au niveau de l'enseignement FLE dans le contexte costaricien, nous n'avons pas trouvé des indices d'une telle fusion avant notre mise en place en 2018.

$\mathrm{Au}$ Costa Rica, l'enseignement du français est aussi présent à l'école primaire publique et dans quelques écoles maternelles publiques et privées. La dynamique de l'enseignement du FLE, dans le milieu de l'éducation publique, reste assez traditionnelle voire stagnée, soit par manque de ressources ou de connaissances soit par l'absence de volonté de la part de l'enseignant. Donc, l'intérêt a été de proposer une séquence pédagogique novatrice avec des matériaux didactiques montessoriens que nos collègues pourront élaborer eux-mêmes.

Nous aborderons à continuation les référents théoriques employés dans la recherche.

\section{Montessori : la méthode de la péda- gogie scientifique}

La méthode Montessori a eu une énorme diffusion dans le monde entier et continue de nos jours à être en vigueur. Le but de la pédagogie est de former l'enfant pour la vie afin qu'il puisse faire face à son entourage. La pédagogue propose une préparation minutieuse de la salle de classe où l'apprenant aura à sa disposition des matériaux sensoriels spécifiques (adaptés aux périodes sensibles) qui leur permettent de travailler de façon autonome. Dans ce sens, l'éducateur est d'abord un observateur. Il est un guide qui accompagne les enfants dans leurs apprentissages et qui les laisse développer leur potentiel.

Montessori a conçu un matériel didactique concret après ses observations auprès des enfants dans une ambiance naturelle : les Casa dei Bambini ${ }^{1}$. 
Elle a permis aux enfants de choisir le matériel qui les intéressait et a éliminé celui qui n'évoquait chez eux aucun intérêt. Progressivement, la pédagogue les a modifiés et les a adaptés à leur besoin. De cette manière, la classe s'est organisée en cinq grands secteurs : la vie pratique, la vie sensorielle, la culture, les mathématiques et le langage. L'enfant est donc libre de choisir le matériel qu'il veut manipuler. Dans ce sens, les activités ne sont jamais imposées par l'enseignant, c'est l'enfant qui décide quelle activité il veut réaliser et il le fait toujours de façon autonome car le matériel permet l'autocorrection. Toutefois, il peut être guidé par l'enseignant quand cela se révèle nécessaire.

Pour la création des activités et du matériel sensoriel, nous nous sommes inspirés des concepts théoriques suivants :

a. Le mouvement : Il est considéré au-delà du point de vue physique. Montessori soutient que le mouvement aide à la construction psychique de l'enfant. C'est un " facteur indispensable dans la construction de la conscience " parce qu'il représente un trait d'union entre l'esprit (MOI) et la réalité extérieure (Montessori, 2016, p.188). Selon l'auteure, la réalité se saisit par le mouvement.

b. Le rythme : Montessori a élaboré un matériel qui demandait des enfants un mouvement rythmique de la main. Elle a vite observé qu'ils étaient capables de répéter le même exercice de nombreuses fois si les conditions étaient réunies, se produisant ainsi une " espèce de mouvement perpétuel ", phénomène que la pédagogue a nommé la "répétition de l'exercice »
(Montessori, 2016, p. 109). De cette manière, l'apprenant atteint de façon naturelle un état de concentration profonde qui lui permet de développer son intelligence et de construire son caractère.

c. La main : La main permet à l'être humain de prendre possession de l'ambiance et de manifester son intelligence. " L’organe moteur qui caractérise l'homme, c'est la main, au service de l'intelligence, pour la réalisation du travail " (Montessori, 2016, p.76). Les petits ont un besoin interne de toucher les objets qui les entourent et de s'en emparer, mais en général, les adultes se mettent à les empêcher cette action naturelle par un besoin de domination. Permettre aux enfants la liberté de toucher et de manipuler les objets signifie aussi de leur permettre de prendre possession de l'ambiance, de la transformer et de manifester son intelligence.

d. Le libre choix : Montessori explique que l'adulte « en voyant les efforts de l'enfant pour exécuter une action souvent inutile ou futile [...] il est tenté de l'aider, interrompant ainsi un travail qui le gêne " (2016, p.83). Dans ce sens, l'adulte devient un obstacle pour le développement de la vie de l'enfant. En effet, en supprimant les aides inutiles de la part des adultes et en laissant les petits choisir par euxmêmes, on leur permet de se développer de façon libre et autonome.

e. Les récompenses et les punitions : Montessori (2016) explique que le fait d'enlever les récompenses systématiques produisent un réveil de la conscience et un sens de la dignité. Selon l'auteure, 
la récompense systématique est inutile -elle donne l'exemple des bonbons -parce qu'elle est de nature à engendrer l'envie et la vanité, au lieu de réveiller une élévation faite d'efforts, d'humilité et de charité. Sous cette perspective, les récompenses sont des punitions puisqu'elles détruisent la joie.

\section{La lecture oralisée comme un travail de sensibilisation à la langue}

Dans le cadre de l'enseignement précoce des langues, le mode d'entrée privilégié pour les petits est de développer leurs capacités d'écoute pour qu'ils puissent distinguer les registres sonores de la langue étrangère (Porcher et Groux, 2003). Les capacités auditives des apprenants doivent s'habituer à la nouvelle langue afin de passer à la compréhension. Pour cette raison, l'usage pédagogique des matériaux authentiques est absolument essentiel.

Puisqu'il s'agit d'un public non-lecteur la lecture doit être à haute voix par autrui, acte qui permet de faciliter l'accès au sens et intègre en même temps l'objet-livre devenant un élément visuel essentiel (Le Bars, 2012). Ceci demande une implication totale de la part du lecteur car celui-ci doit rendre la lecture vivante pour susciter l'attention des enfants et les faire entrer dans le récit progressivement et même les faire participer. Le Bars (2012) insiste sur l'importance de savoir jouer habilement avec son corps : associer le regard, l'audition et la phonation pour capter et maintenir l'intérêt de l'auditeur. Le lecteur doit savoir utiliser l'espace qui lui est offert et faire de la lecture un moment de découverte et de plaisir qui n'est plus vécu seulement comme une activité d'apprentissage.

Pour favoriser l'accès au sens, l'enseignant est censé savoir utiliser les éléments dits " para ou extralinguistiques ». Dans la lecture d'un conte, on dispose de la gestuelle et des images de l'album. Pour raconter une histoire, l'utilisation non seulement des expressions faciales, sinon aussi des mimiques et des mouvements avec les bras et les mains rendent possible la communication efficace du message transmis par l'émetteur. Le geste permet d'améliorer la mémorisation du récepteur et déclenche aussi l'emploi de mots de la part des apprenants. Quant à l'image, Courtillon (2003) signale qu'elle peut apporter deux genres d'informations: L'une de type référentiel (les référents des objets dont on parle) et l'autre de type situationnel (les locuteurs, les lieux et les circonstances de la parole).

Enfin, l'enseignant aide à inférer le sens du vocabulaire, à partir du contexte, de l'assimiler et de le retenir à long terme. C'est le lexique qui constitue le premier contact direct avec la nouvelle langue. Cette inclination naturelle de la part de l'apprenant est exprimée par Cuq et Gruca comme le " réflexe lexical " (2017, p. 365). Cette approche vise alors à saisir le sens petit à petit et cela suppose qu'on comprenne des aspects de la langue sensiblement différents, tels que le lexique et la morphosyntaxe. De cette manière, l'apprenant commence à comparer des mots avec les mots qu'il connaît déjà en langue maternelle afin d'établir des liens entre les deux et donner du sens à un énoncé. Cette recherche de correspondance entre les termes favorise l'appropriation des premières dénotations. 


\section{Méthodologie}

La problématique a été centrée à répondre à la question suivante : Dans quelle mesure des principes de la pédagogie Montessori peuvent-ils mener les enfants non-lecteurs de deux groupes de Grande Section de l'école Saint Benedict à comprendre le lexique du conte "Petit chat perdu " en classe de FLE ?

Dans un premier temps nous avons effectué la révision littéraire pour en tirer des concepts et des principes théoriques de la pédagogie Montessori qui pourraient être utiles dans l'enseignement du français précoce. Ensuite, nous avons conçu une séquence pédagogique de cinq séances comportant des activités variées inspirées de la pédagogie. Avant la mise en pratique, nous avons effectué deux observations non participantes. Les observations participantes ont été faites lors de l'application de la séquence. Finalement, nous sommes passés à l'analyse des résultats et nous avons dégagé les avantages et les inconvénients de la proposition.

Nous avons opté pour une approche qualitative et l'analyse a été de type descriptif. Comme instruments, nous avons utilisé l'interview de l'enseignante de français de l'établissement et les observations non participantes et participantes. Les sujets de l'étude ont été les enfants des deux groupes de la Grande Section l'École Saint Benedict, un établissement privé au Costa Rica où l'enseignement bilingue (espagnol-anglais) est favorisé, mais une leçon de français de 40 minutes par semaine est également donnée. Pour la collecte des données nous avons employé une grille d'observation et un journal de bord (Voir annexe 1).
Pour chaque séance, nous avons élaboré une fiche pédagogique dans laquelle nous avons établi des objectifs et un principe Montessori à favoriser. Les activités mises en place correspondent à des activités propres de la méthode Montessori adaptées à la didactique du FLE. Par rapport au matériel sensoriel, celui-ci a été élaboré par nous-mêmes.

Comme document authentique, nous nous sommes appuyés sur le conte pour enfants intitulé « Petit Chat Perdu " de la série des albums du Père Castor de la maison d'éditions Flammarion (Voir annexe 2). Le contenu linguistique du document est assez simple et suffisamment riche dans le champ lexical à exploiter. Cependant, l'ouvrage date du 1997 et les images qui y apparaissent étaient (à nos yeux) un peu trop démodées. C'est pourquoi, nous avons décidé de le faire réillustrer et de l'imprimer en grand format (Voir annexe 3). Nous avons ainsi pris cette décision non seulement pour un choix purement esthétique ; mais nous avons pris en considération les principes théoriques postulés par Vanthier qui affirme que l'une des caractéristiques souhaitables dans le choix d'un album à utiliser en classe est " d'avoir un format qui se prête à une lecture collective (suffisamment grand pour que les illustrations soient visibles par tous) et présenter une dimension esthétique au niveau de la langue et l'illustration. » (2010, p. 63)

L'objectif de la séquence a demandé aux enfants d'être capables de comprendre de façon générale, le conte entendu en français et de le reproduire en langue maternelle à la fin de celle-ci, tout en apprenant le lexique des animaux de la ferme, leur nourriture et quelques structures grammaticales présentes dans le récit. 


\section{Résultats}

Tout d'abord, le fait d'avoir travaillé en îlots pour la plupart d'activités nous a permis de suivre de plus près le progrès des enfants tout au long de la séquence et nous a facilité la gestion de la classe. En effet, nous avons constaté que les apprenants étaient très habitués à suivre une conduite spécifique : rester calmes, ne pas bouger, avoir une place fixe, etc. Le calme et le silence rassurent l'adulte et il nous a fallu des efforts considérables pour éviter de tomber dans le même comportement. Le fait de pouvoir utiliser le mouvement du corps pour apprendre a suscité en eux du bonheur et ils ont vite adopté le droit de circuler librement dans la classe.

En ce qui concerne la discipline, la théorie nous signale que celle-ci ne doit pas être imposée aux enfants parmi des récompenses ou des punitions. Nous avons constaté que les apprenants étaient habitués aux gestes de gratification pour leur mérite car ils demandaient à chaque fois si un prix allait être donné. Toutefois, ils sont bien arrivés à s'investir dans les activités et à en prendre du plaisir, oubliant très vite que le prix était inexistant.

Quant à l'autonomie, les apprenants ont démontré d'être capables de diriger leur propre apprentissage. Puisqu'il s'agit de l'apprentissage d'une langue étrangère, nous avons considéré que chaque exercice devrait être dirigé par chaque stagiaire, mais nous avons constaté qu'une fois la dynamique comprise, les apprenants voulaient la diriger par eux-mêmes. Nous leur avons donné la place de le faire car ils évoquaient leur autonomie. Ils étaient fiers de pouvoir agir et prouver comment la compréhension du vocabulaire était déjà acquise et comment ils voulaient bien prononcer les mots et les structures qu'ils étaient en train d'apprendre.

La correction de l'exercice n'a pas été nécessaire car il ne fallait que le répéter à plusieurs reprises pour que l'apprentissage fût accompli. La correction a été faite aux moments où l'agir des enfants provoquait un obstacle à l'apprentissage des camarades ou lorsqu'il s'agissait d'une situation qui représentait en quelque sorte un danger pour leur sécurité.

Par rapport aux principes de la théorie, ceux-ci ont inspiré les activités, mais nous les avons également adoptés dans notre attitude tout au long de la séquence. Pour cette raison, nous avons donné aux apprenants l'espace de parler, de participer, d'expérimenter avec le matériel et de s'exprimer en respectant leur individualité.

Concernant la langue, nous devons rappeler que le français constitue la deuxième langue étrangère des élèves. Par conséquent, cela a été normal de voir le recours immédiat à l'anglais au moment de donner une réponse ou de trouver une correspondance d'un mot. Cependant, nous trouvons que cela a été avantageux car les stratégies d'apprentissage d'une langue étrangère étaient déjà adoptées. En ce qui concerne l'usage de la langue maternelle, nous avons, à tout moment, essayé d'utiliser la langue française. Cependant, nous avons fait appel à l'espagnol dans certains cas où nous devions faire des remarques par rapports aux comportements non-désirables, notamment au moment d'intervenir dans une dispute.

Quant à l'élaboration du matériel, il faut mentionner que cela implique un investissement considérable de la 
part de l'enseignant. Cela pourrait éventuellement constituer une difficulté selon les ressources étant à disposition dans chaque établissement. Par contre, le coût pourrait être baissé si l'on se sert des matériaux recyclables et simplifiés au niveau esthétique.

Enfin, nous trouvons que dès la première lecture du conte, le récit général a été bien saisi. Les enfants ont su reconnaître les personnages en français et l'idée général de l'histoire. La deuxième lecture et les activités proposées par la suite ont eu comme objectif d'apprendre aux enfants le champ lexical du conte (les animaux de la ferme et les aliments) ainsi que les structures simples qui se répétaient tout au long du récit. Même si la production orale n'a pas été envisagée dans cette séquence, nous avons constaté que les enfants ont été capables de reconstruire le conte en espagnol et plusieurs enfants ont même utilisé les structures apprises en français en mélangeant ses productions avec la langue maternelle.

\section{Conclusions}

Face à l'absence d'études de l'application de la méthode Montessori pour l'enseignement d'une langue étrangère, notamment le français, nous considérons que cette recherche peut apporter une nouveauté au domaine du FLE au Costa Rica.

Grâce aux séances menées, nous avons pu réfléchir sur l'importance de varier les supports en nous inspirant des pédagogies alternatives, afin de diversifier le cours de FLE et d'évoquer chez les petits, le goût d'apprendre, ce qui est essentiel à un court âge où les apprentissages peuvent se révéler plus critiques que dans d'autres périodes de leur développement. À cet effet, la pédagogie Montessori a constitué une source d'inspiration et un outil très puissant pour permettre aux enfants d'avoir une expérience d'apprentissage unique.

Nous avons également constaté que l'utilisation d'un conte avec les petits est un travail qui implique une préparation exhaustive avant sa mise en place. En effet, le choix d'un tel document doit répondre à des éléments divers comme les objectifs linguistiques, les intérêts des apprenants, ainsi que leurs besoins d'apprentissage. De même, le paratexte joue un rôle prépondérant spécialement dans le travail d'éveil à la langue. Dans notre cas, nous avons vérifié que l'utilisation des images claires et esthétiques, accompagnant le document ainsi que la gestuelle, constituent des outils d'une énorme importance pour favoriser l'accès au sens chez les apprenants. Plus les supports didactiques sont visuellement coordonnés, plus l'enfant aura envie de les manipuler.

Nous nous sommes intéressées à la formation holistique de nos élèves, surtout si nous prenons en compte l'âge de notre public cible, car ils traversent une étape critique dans la consolidation de leur caractère et leur estime de soi. Nous avons ainsi considéré que l'approche Montessori est un outil qui va au-delà du travail de la langue, et elle se présente comme une opposition à l'automatisation qui persiste souvent dans l'éducation traditionnelle. Dans le contexte actuel, il est important d'expérimenter avec des nouvelles voies d'enseignement afin d'enrichir les dynamiques d'apprentissage dans 
le cours du FLE.

\section{Note}

1. En 1907, Maria Montessori ouvre la première maison d'enfants ou Casa dei Bambini dans une banlieue ouvrière de Rome. Elle a remarqué que plusieurs enfants passaient la journée seuls et circulaient libres dans les immeubles car les parents partaient travailler. Dans la Casa dei Bambini, Montessori mènera ses expérimentations pour développer sa méthode qu'elle nommera "la pédagogie scientifique ». Elle a fait élaborer des meubles adaptés à la taille des enfants et des matériaux de sa propre conception. Elle leur a donné la liberté d'explorer ce nouvel environnement, de choisir et de prendre les objets avec lesquels ils souhaitaient travailler.

\section{Bibliographie}

Album. (2017). Lexicographie cnrtl.fr. Nancy, France : CNRS / Nancy Université. www.cnrtl.fr. Récupéré de : http://www.cnrtl.fr/definition/album

Assie, G. et Kouassi, R. (s.d) Cours d'initiation à la méthodologie de recherche. Abidjan : École pratique de la chambre de commerce et de l'industrie-Abidjan. Récupéré de : http://www.dphu.org/uploads/attachements/books/books_216_0.pdf

Baribeau, C. (2005) Le journal de bord $\mathrm{du}$ chercheur. L'instrumentation dans la collecte de données. Recherches Qualitatives - Hors-Série (2), p. 98-114. Récupéré de : http:// www.recherche-qualitative.qc.ca/ revue/les-collections/hors-serieles-actes/

Bernicot, J. et Bert-Erboul, A. (2009).
L'acquisition du langage par l'enfant. Éditions In Press.

Bertocchini, P. et Constanzo, E. (2008). Manuel de formation pratique pour le professeur de FLE. CLE International.

Boniface, C. (2004). Aimer lire : Guide pour aider les enfants à devenir lecteurs. Bayard.

Bouve, J. (2016). Les rituels scolaires. (Mémoire de maîtrise, École supérieure du professorat et de l'éducation, Académie de Lille) Récupéré de : https://dumas.ccsd.cnrs. fr/dumas-01491538

Brauer, B. (2017). Apprentissage de la langue dans la pédagogie Montessori. Récupéré de : https://www. association-montessori.lu/apprentissage-de-langue-pedagogie-montessori/

Castejón Costa, J.L. (2016) Promoción del lenguaje oral en niños preescolares a través de la lectura dialógica de cuentos. Psicología y Educación: Presente y Futuro. ACIPE- Asociación Científica de Psicología y Educación. Récupéré de : http://hdl. handle.net/10045/63648

Conseil de l'EUROPE. (2001). Un cadre européen commun de référence pour les langues : apprendre, enseigner, évaluer. Didier. Récupéré de : https://www.coe.int/fr/web/ common-european-framework-reference-languages

Courtillon, J. (2003). Élaborer un cours de FLE. Hachette.

Cuq, J.P. et Gruca, I. (2017). Cours de didactique du français langue étrangère et seconde. PUG.

Delgado, J. (s. d.) The Calming Jar: A technique that all parents should know. Developmental psychology. Récupéré de : https://psychology- 
spot.com/calming-jar-maria-montessori/

Dufour, N. (2013). L'usage du geste au sein de la classe de langue vivante étrangère. Education. Récupéré de : https://dumas.ccsd.cnrs.fr/dumas-00868482/document

Eissler, T. (2013). Montessori, c'est fou! École. Vivante

Ferrière, A. (1922). L'activité spontanée chez l'Enfant. Éditions Internationales Populaires.

Hainstock, E. (1986). The Essential Montessori. Updated Edition. Plume.

Horth, L. (2004). La concordance entre l'approche Montessori et le Programme de formation de l'école québécoise. Résultat d'une analyse documentaire. Québec : Ministère de l'Éducation du Québec. Récupéré de : http://www.education. gouv.qc.ca/references/publications/ resultats-de-la-recherche/detail/ article/la-concordance-entre-lapproche-montessori-et-le-programme-de-formation-de-lecole-quebecoise-resu/

Lawrence, L. (2001). Ayude a sus hijos a leer y escribir con el método Montessori. Ediciones Paidós, Ibérica, S.A.

Le Bars, M. (2012). Les enfants et la lecture : mettre en voix pour amener au plaisir de lire. (Mémoire de Master 2, Université Stendhal, Grenoble, France). Récupéré de : https://dumas.ccsd.cnrs.fr/dumas-00735981/ document

Lincoln, Y.S. et Guba, E.G. (1985). Naturalistic Inquiry. Sage.

Littérature pour la jeunesse (2017). Dictionnaire Larousse. Récupéré de : http://www.larousse.fr/encyclopedie/divers/litt\%C3\%A9rature_ pour_la_jeunesse/63254\#905409

Loubet des Bayle, J.-L. (2000) Initia- tion aux méthodes des sciences sociales. L'Harmattan. Récupéré de : http://classiques.uqac.ca/contemporains/loubet_del_bayle_jean_louis/ initiation_metho_sc_soc/initiation_ metho_sc_soc.html

Margaux, M. (2015). La pédagogie Montessori à l'école de la République. (Mémoire de maîtrise, Université de Nantes, France). Récupéré de : http://dune.univ-angers.fr/documents/dune 4940

Martineau, S. (2005). L'observation : enjeux, possibilités et limites. L'instrumentation dans la collecte de données. Recherches Qualitatives. Hors-Série (2), p. 5-17. Récupéré de : http://www.recherche-qualitative.qc.ca/revue/les-collections/ hors-serie-les-actes/

Mebarki, M. (2015). Les étapes de la compréhension de l'oral en FLE. (Mémoire de maîtrise, Université Mohamed Khider - Biskra). Récupéré de : http://dspace.univ-biskra.dz:8080/jspui/ bitstream/123456789/8171/1/MEBARKI\%20Maroua.pdfConsulté [en ligne] le 2 novembre 2017

Médioni, M-A. (2011). Enseigner la grammaire et le vocabulaire en langues. Chronique sociale.

Méndez, M. (2009). Louis XIV, Charles Perrault et la naissance de la littérature pour enfants. Revista de Lenguas Modernas. Volumen 11.

Miles, M.B. et HUBERMAN, A.M. (2003). Analyse des données qualitatives. (2ème éd.). De Boeck.

Monés, J. et Vallet, M. (2000). Pedagogías del siglo XX. Editorial Cisspraxis, S.A.

Montessori, M. (1911). El método de la pedagogía científica aplicado a la educación de la infancia en las 
"Case dei Bambini". Ramón de S.N araluce.

Montessori, M. (1917) Le décalogue de l'éducateur. Récupéré de : https:// www.association-montessori.lu/ maria-montessori/decalogue-deleducateur/Consulté [en ligne] le 7 novembre 2017

Montessori, M. (2010) Éducation pour un monde nouveau. Éditions Desclée de Brouwer.

Montessori, M. (2012). The Montessori Method Scientific Pedagogy as Applied to Child Education in 'The Children's Houses'. Traduit par Goeorge A.E. Ouvrage du domaine public.

Montessori, M. (2016). L'enfant. Éditions Desclée de Brouwer.

Montessori, M. (2016). Le manuel pratique de la méthode Montessori. Traduit par Poussin C. Éditions Desclée de Brouwer.

Montessori, M. (2017). Les écoles Montessori en France et dans le monde. Récupéré de : http://montessorilandes.org/ecoles-montessori-france-monde/ Natacha. (1997). Petit chat perdu. Albums du Père Castor. France : Éditions Flammarion.

Porcher, L. et Groux D. (2003) L'apprentissage précoce des langues. Vendôme. Presses Universitaires de France.

Proscolli, A. et Anastassiadi, M.C. et al (2007). Enseigner le français langue étrangère à l'école primaire: méthodes et pratiques. Université Nationale et Capodistrienne d'Athènes, Faculté des Lettres. Récupéré de : http://www.frl.uoa.gr/fileadmin/frl. uoa.gr/uploads/sinedria/Actes_Primaire_2007.pdf

Riverin-Simard, D. (1997). Positions paradigmatiques et recherches sur le développement vocationnel adulte. Cahiers de la recherche en éducation (4), p.59-91. Récupéré de : http://www.fse.ulaval.ca/danielle. riverin-simard/pdf/positions_paradigmatiques.pdf

Robert, J-P. (2008). Dictionnaire pratique de didactique du FLE. Ophrys.

Rubio, C. (2015). La escuela innovadora de Carmen Lyra. La Nación. Récupéré de : https://www.nacion.com/ viva/cultura/la-escuela-innovadora-de-carmen-lyra/P7ZQBWJBDBGBPI4TB3GZ5EUBRE/story/

Sampieri, R. (2003). Metodología de la Investigación. McGraw-Hill Interamericana Editores, S.A.

Standing, E. M. (1991). La Revolución Montessori en la Educación. Siglo XXI Editores S.A.

Tellier, M. (2008) Dire avec des gestes. Le Français dans le monde. Recherches et applications, Français dans le monde. Récupéré de : https://hal.archives-ouvertes.fr/ hal-00371029/PDF/Dire_avec_des_ gestes_FDLM_2008.pdf

Tréville M-C. et Duquette L. (1996) Enseigner le vocabulaire en classe de langue. Hachette.

Trilla, J et Cano, E. (2002). El legado pedagógico del siglo XX para la escuela del siglo XXI. Graó.

Tourret, L. et Quenehen, M. (Production). (2017, 24 septembre). France Culture. Montessori Superstar [Podcast audio]. Récupéré de : https:// www.franceculture.fr/emissions/ rue-des-ecoles/montessori-superstar

Van Campenhoudt, L. et Quivy Raymond (2011). Manuel de Recherche en Sciences Sociales. Dunod.

Vanthier, H. (2010). L'enseignement aux enfants en classe de langue. Paris: CLE International. 
Vincent, F. (2010). Les rituels en Grande Section. Cahiers pédagogique (479) : Dossier Les apprentissages fondamentaux à l'école primaire. Récupéré de : http://www.cahiers-
pedagogiques.com/Les-rituels-enGrande-Section

Zapata, A. (2013). L'évolution de l'enseignement du vocabulaire dans la classe de L2. Revista de 
Lenguas Modernas (19), p. 437-447.

\section{Annexes}

\section{Grille d'observation non participante}

Universidad de Costa Rica

Guide d'observation d'un cours régulier en FLE de la Grande Section École Saint Benedict

Professeur:

Heure:

Groupe:
Date:

Durée de la séance:

Nombre d'élèves:

1. Avant la séquence :

Décrivez de manière globale le contexte de la classe: ambiance, attitude des élèves, motivation vis-à-vis du cours de français, etc.

Décrivez la disposition de la classe: surface, nombre de pupitres, décoration, illumination, accès à la technologie, etc.

2. Pendant la séquence :

a) Clarté sur les consignes.

b) Cohérence entre l'objectif pédagogique et l'application de la séquence.

c) Rôle des apprenants (actifs, passifs, motivés, indifférents, etc.).

d) Disposition physique des apprenants pendant les activités proposées par le professeur.

e) Quelle a été la réaction de la part des apprenants (intéressés, ennuyés, actifs, passifs, etc.)

f) Parlez du rôle de l'enseignant au cours de la session (interaction, questions posées, etc.).

g) Usage de la langue maternelle de la part du professeur.

h) Supports didactiques utilisés.

3. Après la séquence :

a) Pensez-vous que l'application de la séquence didactique a été efficace ? Expliquez pourquoi.

b) Citez deux possibles aspects à améliorer.

c) Citez les points forts de la séquence pédagogique mise en place par le professeur.

4. D'autres informations :

a) Commentaires du professeur. 
b) Tout commentaire additionnel que vous considérez nécessaire.
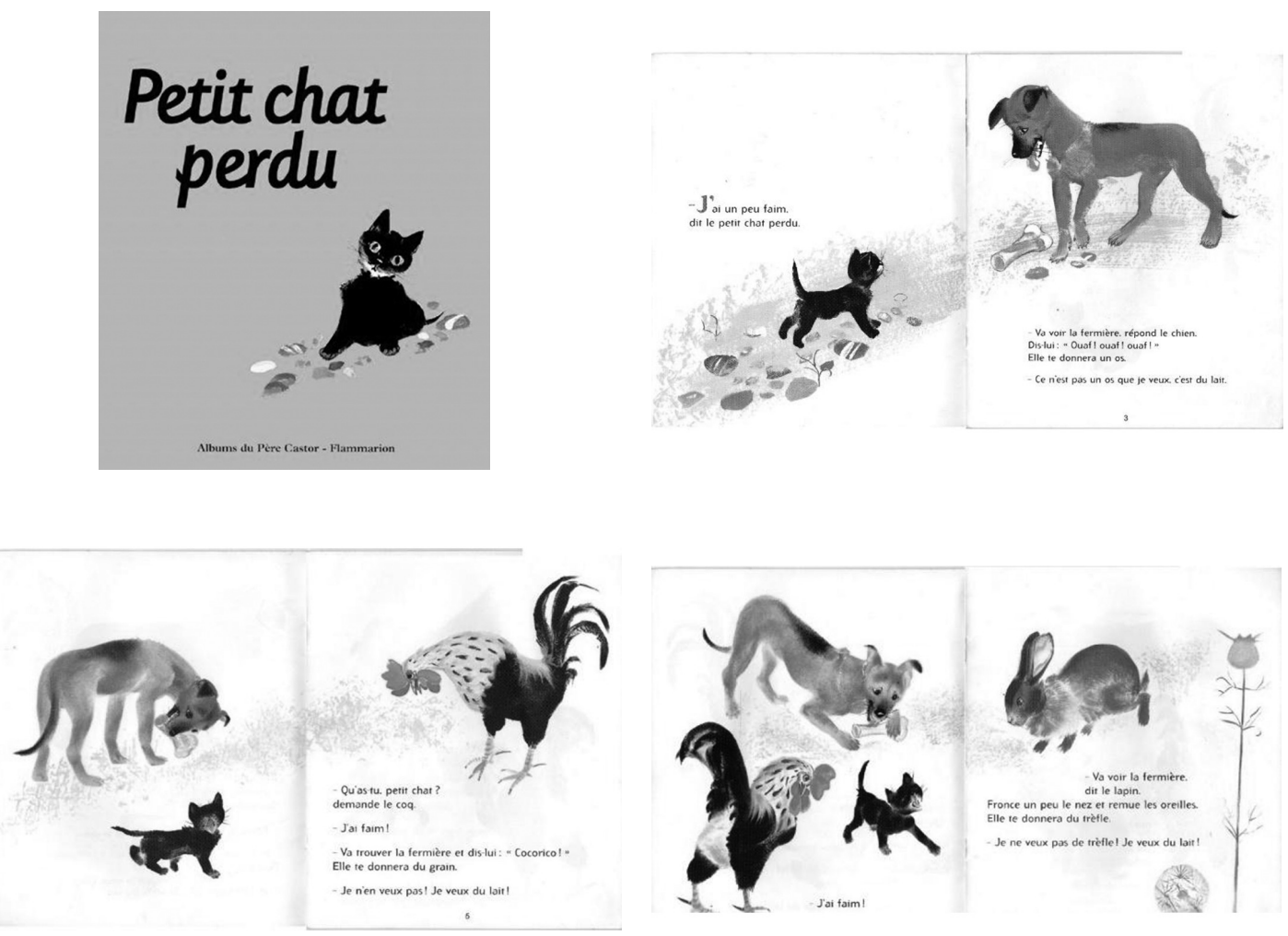

5. Extrait du conte « Petit chat perdu » édition 1997 : Albums du Père Castor.
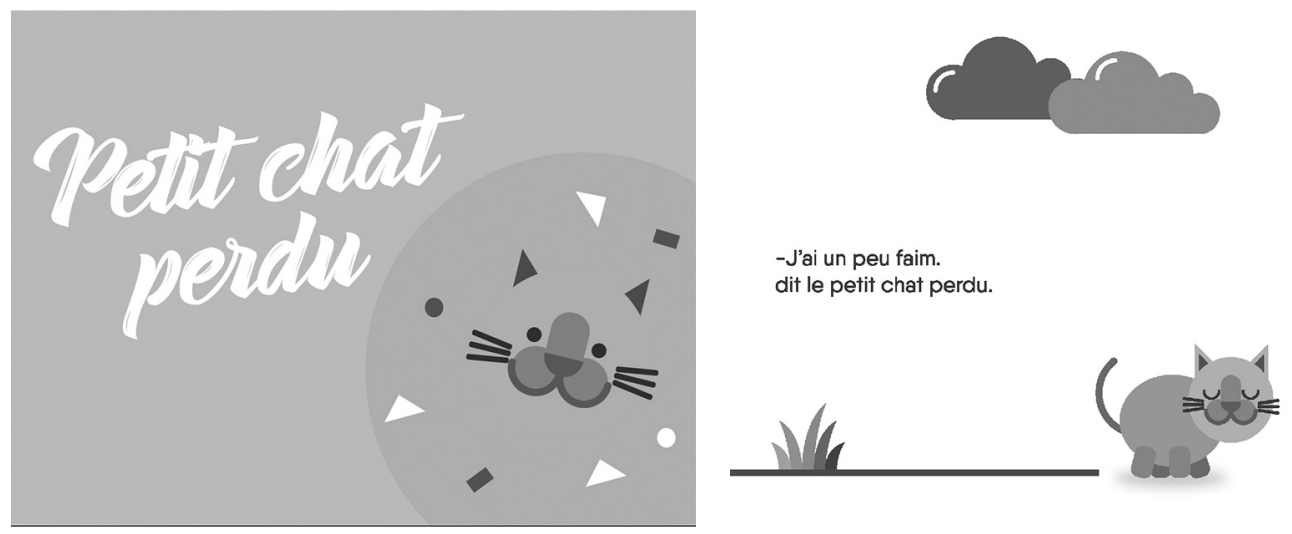
6. Extrait de la version réillustré du « Petit chat perdu » par Carlos Alfaro.

- Va voir la fermière, répond le chien. Dis-lui: "Ouaf! ouaf! ouaf!"

Elle te donnera un os.

- Ce n'est pas un os que je veux, c'est du lait.
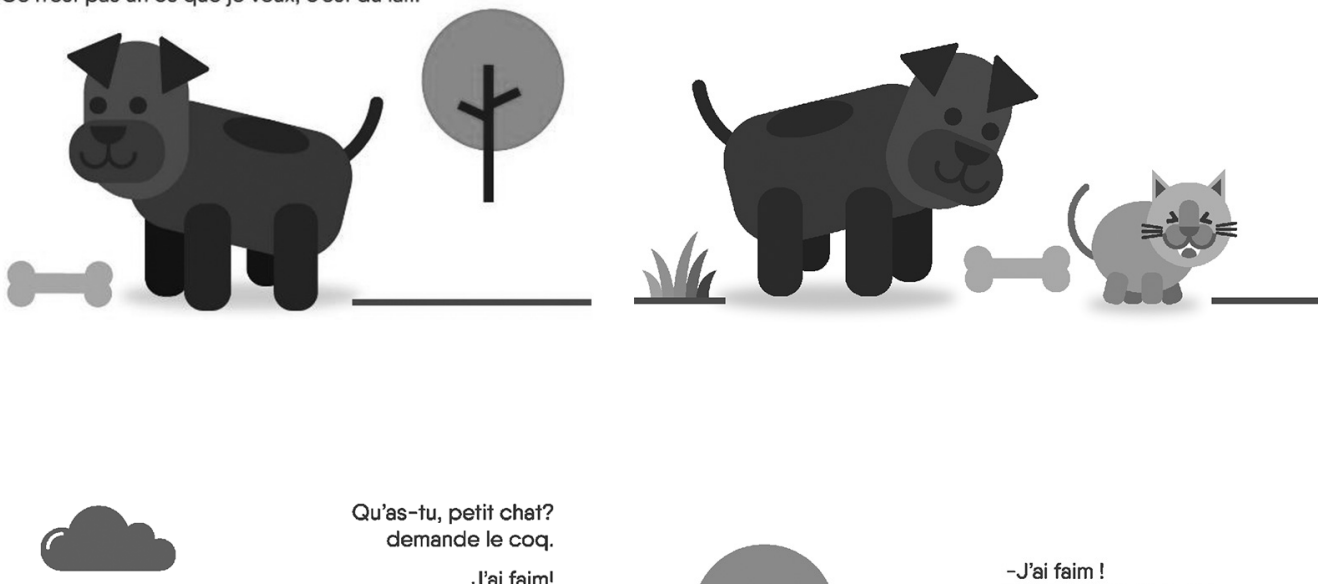

Qu'as-tu, petit chat? demande le coq.

J'ai faim!

Va trouver la fermière et dis-lui : "Cocorico ! "

Elle te donnera du grain.
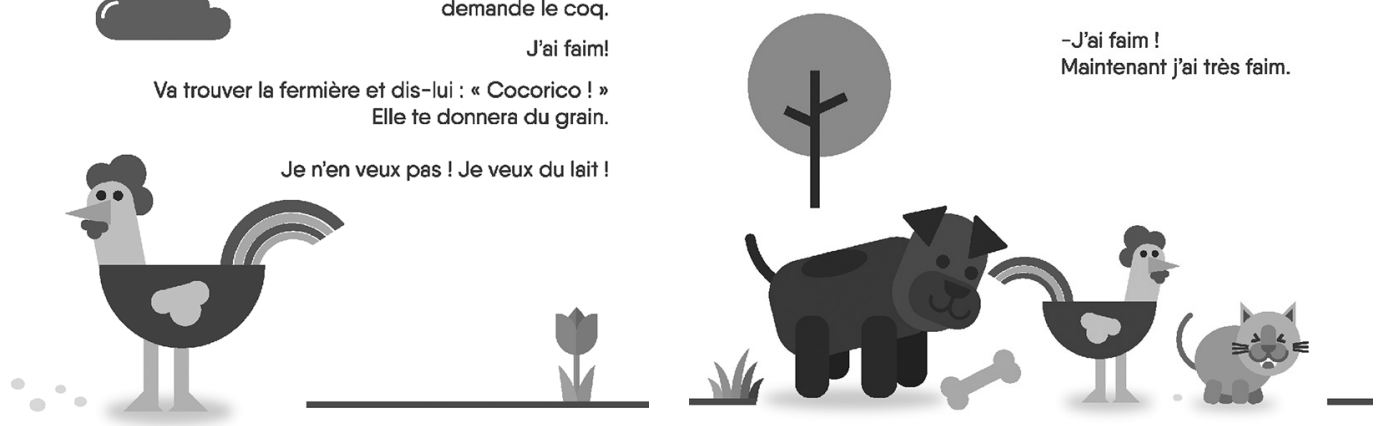
